\title{
Retraction Note: Land subsidence in Beijing from 2017-2018 revealed by Sentinel-1A TOPS time series interferometry
}

Peilian Ran, Shaoda Li, Keren Dai and Xiaoxia Yang

Retracted article: E3S Web of Conferences 218, 04002 (2020)

https://doi.org/10.1051/e3sconf/202021804002

The Guest Editors and the Publisher have retracted this article. This paper has been formally retracted from publication by the authors on ethical grounds. Actually, one of the authors (Mr. Peilian Ran) neglected that a similar paper was already presented and published in a previous conference proceedings. The authors are responsible for this mistake and apologise for it. Request approved by the proceedings Editor and the Publisher on February 2, 2021. 